$16^{\text {th }}$ International Conference on

AEROSPACE SCIENCES \& AVIATION TECHNOLOGY,

ASAT - 16 - May 26 - 28, 2015, E-Mail: asat@ mtc.edu.eg

Military Technical College, Kobry Elkobbah, Cairo, Egypt

Tel : +(202) 24025292 - 24036138, Fax: +(202) 22621908

\title{
A Rapid Gun Firing Correction Algorithm Using Discrete Time Transfer Matrix Method
}

\author{
Mostafa Khalil", Xiaoting Rui** and Hossam Hendy*
}

\begin{abstract}
To improve the next shot hit accuracy, a firing angle correction algorithm will be developed using the distance error computed between the impact and target coordinates to determine the launch azimuth and quadrant elevation angles for artillery projectiles. This correction method will be developed using the discrete time transfer matrix method of the projectile modified point mass model DTTMM-4DOF to improve correction method performance. To validate the proposed algorithm, a spin-stabilized $155 \mathrm{~mm}-\mathrm{HE}$ projectile flight trajectory will be used, assuming that wind magnitude and direction with the down range are changing as function of projectile altitude.
\end{abstract}

Keywords: Firing correction; Un-guided projectile; 4-DOF; Discrete Time Transfer Matrix; 105mm-HE; 155mm M107.

\section{Introduction}

In case of unguided projectiles, to improve the weapon's accuracy [1], the error between the projectile impact point and target has to be estimated precisely to evaluate the weapon's accuracy and hence; an appropriate projectile firing correction is done based on the first shoot impact error to increase the next shoot accuracy. Traditionally, firing tables is used to calculate the projectile standard firing data needed to hit a desired target and also compute the firing data corrections in case of non-standard flight conditions [2]. These nonstandard flight conditions are resulted from variances in many parameters such as meteorological data, projectile mass properties, propellant temperature, and altitude difference between the launch point and the target [3]. To improve the weapon's hit accuracy, these nonstandard flight conditions have to be predicted accurately as well as possible especially the meteorological data [4], [5]. Using lookup search, all firing data can be determined automatically using a stored tabulated data for both standard and non-standard conditions. To speed up the searching process, different graphical tools such as graphical firing tables, graphical site tables, and wind cards, are used but with low accuracy due to simplifications [2], [6].

\footnotetext{
Egyptian Armed Forces, Egypt; mostafakhalil80@gmail.com .

** Professor, Institute of Launch Dynamics, Nanjing University of Science and Technology.
} 
In order to improve the performance of the fire control system, it is necessary to develop an accurate and fast correction technique for projectile external ballistics problems. Currently, firing correction methods are divided mainly into two kinds: (1) firing table approximation; and (2) numerical methods. Due to the fast developments of computer technologies, numerical methods are widely used as the calculation processes become easier and general for any kind of gun, however, firing table approximation is different for different gun type and different firing conditions.

\section{Related Work}

Many researchers developed different algorithms to improve the impact accuracy. In 1996, a mathematic model for determining firing data by computations considering the Coriolis effect and the firing site and target are not in the same level [7] was developed. In 1997, a weighted function model based on small perturbation assumptions for all measurable real shooting conditions was developed to estimate the correction angle required to improve impact accuracy [8]. In 2001, a firing angle iterative range correction method for tank fire system was developed based on the point mass model [9]. Another correction method used in ship-borne gun fire system has been developed to calculate the fire data against the targets on shore [10]. In 2003, a model to calculate the firing data and flight time with the target position has been developed by solving the projectile external ballistic equations once only to reduce processing time, as well as methods to correct the informal ballistic and meteorological conditions, movement of the ship and the side deviation of projectiles were presented [11]. In 2007, Harlin and Cicci developed a state transition matrix STM to make corrections to ballistic missile initial state vector to achieve predefined final state vector using a 6DOF model [12]. In 2011, a differential algorithm with two height levels was developed to accurately calculating the ahead hit position for real antiaircraft gun system [13].

In flight control systems using iterative method, the solution of azimuth and quadrant elevation is determined through iterations of trajectory simulation in nonstandard conditions instead of searching firing data on the pre-calculated tabulated data in standard condition and then, applying corrections to compensate the corresponding nonstandard conditions [3], [14]. NATO Armaments Ballistic Kernel (NABK) is one of the most notable system uses an iterative method to compute the firing data [15], where the ballistic trajectory model used during iterations is for the modified point mass model (4-DOF) [16] or five-degrees-offreedom model [17]. Many algorithms were developed to insure the firing data convergence during iterative trajectory simulations [3], [18]. These iterative methods require an efficient algorithm to minimize the computation time. 


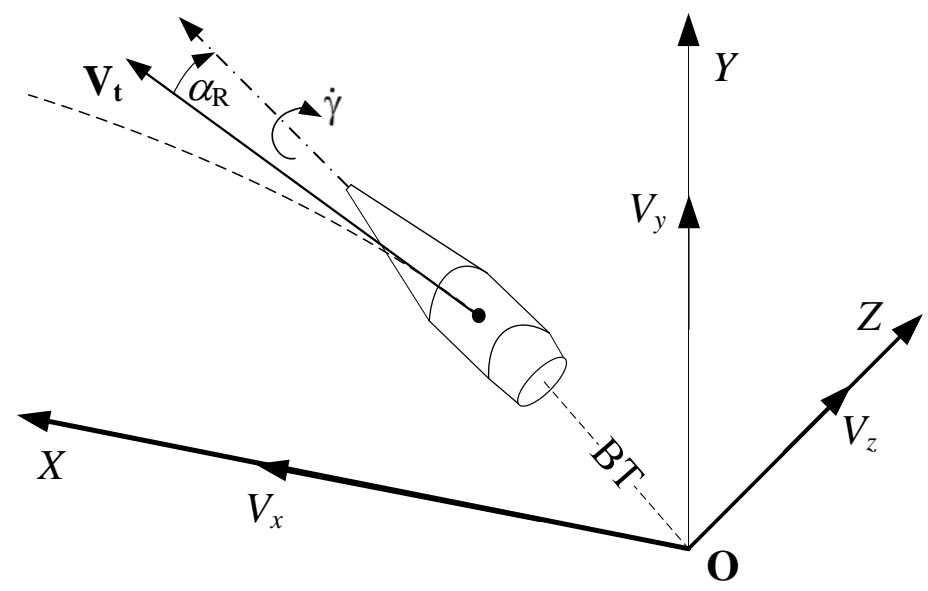

Fig. 1 Coordinate system and directions of ballistic target

\section{Projectile Trajectory model}

The projectile modified point mass equations of motion 4-DOF with respect to earth fixed coordinate system $(X, Y, Z)$ as shown in Fig. 1 are given by $[17,19]$

$$
\begin{gathered}
\dot{\mathbf{X}}=\boldsymbol{v}=\left[\begin{array}{lll}
\dot{x} & \dot{y} & \dot{z}
\end{array}\right]^{T}=\left[\begin{array}{lll}
v_{x} & v_{y} & v_{z}
\end{array}\right]^{T} \\
\dot{\boldsymbol{v}}=-\rho\left|\boldsymbol{v}_{r}\right| a_{1} C_{x} \boldsymbol{v}_{r}+\rho a_{1} C_{y}^{\prime}\left|\boldsymbol{v}_{r}\right|^{2} \boldsymbol{\alpha}_{\mathrm{R}}-\rho a_{2} C_{z}^{\prime \prime} \dot{\gamma}\left[\boldsymbol{v}_{r} \times \boldsymbol{\alpha}_{\mathrm{R}}\right]+\mathbf{g}+\boldsymbol{\Lambda} \\
\ddot{\gamma}=-\frac{\rho . s . l^{2}\left|\boldsymbol{v}_{r}\right|}{2 C} \dot{\gamma} \cdot m_{x D}^{\prime} \\
\boldsymbol{\alpha}_{\mathrm{R}}=\frac{2 . C \cdot \dot{\gamma}}{\rho . s . l \cdot\left|\boldsymbol{v}_{r}\right|^{4} m_{z}^{\prime}}\left[\mathbf{g} \times \boldsymbol{v}_{r}\right] \\
\boldsymbol{v}_{\boldsymbol{r}}=\boldsymbol{v}+\boldsymbol{w}
\end{gathered}
$$

Where, $C_{x}, C_{y}^{\prime}, C_{z}^{\prime \prime}, m_{x D}^{\prime}$ and $m_{z}^{\prime}$ are the aerodynamic drag force, lift force, Magnus force, spin damping and pitching moments coefficients respectively; $v$ is the projectile velocity vector in earth fixed coordinate; $\left|v_{r}\right|$ is the projectile total velocity vector with respect to air; $\rho$ is the air density; $m, C, A, l$, and $s$ are the projectile mass, axial and transverse moments of inertia, reference length and reference area respectively; $\alpha_{R}$ is the projectile angle of repose; $\boldsymbol{w}$ is the wind velocity vector in earth fixed coordinate; and the earth's gravitational $\mathbf{g}$ and Coriolis $\Lambda$ accelerations are given by $[17,18]$

$$
\begin{gathered}
\mathbf{\Lambda}=2 \Omega\left[\begin{array}{ccc}
0 & -\cos \mu \sin \lambda & -\sin \mu \\
\cos \mu \sin \lambda & 0 & \cos \mu \cos \lambda \\
\sin \mu & -\cos \mu \cos \lambda & 0
\end{array}\right]\left[\begin{array}{l}
V_{x} \\
V_{y} \\
V_{z}
\end{array}\right] \\
\mathbf{g}=-g \cdot\left[\begin{array}{ccc}
\frac{X_{1}}{R_{e}} & 1-\frac{2 X_{2}}{R_{e}} & 0
\end{array}\right]^{T}
\end{gathered}
$$


Where, $g=g_{o} \cdot[1-0.0026 \cos (2 \mu)], g_{o}=9.80665 \mathrm{~m} / \mathrm{s}^{2} ; \Omega$ is the earth's angular velocity; $\mu$, $\lambda$ are the corresponding latitude and longitude of the firing sight; and $R_{e}$ is the average radius of the earth $(=6356766 \mathrm{~m})$.

Due to spherical earth approximation, the instantaneous projectile altitude $H$ is defined as [17]

$$
H=X_{2}+\frac{X_{l}^{2}}{2 . R_{e}}
$$

\section{Firing Correction Algorithm}

For a given projectile ballistic trajectory with impact point $\mathbf{X}_{\mathrm{P}}=\left\{X_{\mathrm{P}}, Y_{\mathrm{P}}, Z_{\mathrm{P}}\right\}$, the error between this impact point and the target $\mathbf{X}_{\mathrm{T}}=\left\{X_{\mathrm{T}}, Y_{\mathrm{T}}, Z_{\mathrm{T}}\right\}$, as mentioned in Eq. (9), has to be minimized to improve the weapon's accuracy. In case of unguided projectiles, $\Delta \mathbf{X}$ is the distance error to be iteratively compensated by correcting one or more initial flight parameters. These adjustments could be the initial position, velocity, or projectile attitude. Based on discrete time transfer matrix method DTTMM-4DOF based on projectile modified point mass model stated in ref. [21], a two-dimensional iterative method is developed to obtain the projectile launching angle for desired target distance and direction.

The proposed algorithm assumed that the only known elements of the impact state is the distance error $\Delta \mathbf{X}$, and the parameter to be corrected to compensate this error is the projectile launch angle $\mathrm{QE}$ and $\mathrm{AZ}$, where the projectile muzzle position vector and velocity magnitude are assumed constants. This two dimensional angle is consist of quadrant elevation angle QE which is controlling the distance between launching point and the target and azimuth angle $\mathrm{AZ}$ which determines the firing direction. The main advantage of this method is the ability of computing the range derivative with respect to $\mathrm{QE}$ during computations of projectile reference trajectory including non-standard conditions, where the pre-calculated and stored derivative may become inaccurate in some non-standard conditions [3].

The projectile state error $\Delta \mathbf{S}$ is comprised of 7 elemental errors with the projectile reference trajectory, which are the projectile position, velocity, and spin rate respectively as mentioned in $\mathrm{Eq}(10)$.

$$
\begin{gathered}
\Delta \mathbf{X}=\mathbf{X}_{\mathrm{P}}-\mathbf{X}_{\mathrm{T}} \\
\Delta \mathbf{S}_{7 \times 1}=\left[\begin{array}{lllllll}
\Delta x & \Delta y & \Delta z & \Delta v_{x} & \Delta v_{y} & \Delta v_{z} & \Delta \dot{\gamma}
\end{array}\right]^{T}
\end{gathered}
$$

To set a direct relation between the state errors $\Delta \mathbf{S}$ during any two successive time step, due to deviation of one or more state parameters for the reference trajectory, a state correction matrix $\boldsymbol{\Phi}$ is deduced using a modified Jacobian $\overline{\mathbf{J}}$ of the system mentioned in ref. [21] as

$$
\begin{gathered}
\Delta \mathbf{S}^{t_{i+1}}=\boldsymbol{\Phi}_{7 \times 7}^{t_{i}} \cdot \Delta \mathbf{S}^{t_{i}} \\
\boldsymbol{\Phi}_{7 \times 7}^{t_{i}}=\mathbf{I}_{7}+\overline{\mathbf{J}}_{7 \times 7}^{t_{i}} \cdot \Delta t
\end{gathered}
$$




$$
\overline{\mathbf{J}}=\mathbf{J}_{7 \times 13} \cdot \mathbf{J}_{\Delta \boldsymbol{\alpha}} \cdot \mathbf{J}_{\Delta \boldsymbol{w}}
$$

The Jacobian matrix $\mathbf{J}_{7 \times 13}$, contains the partial derivatives of projectile position and velocity vectors and spin rate w.r.t. all state variables in Eq. (10), as

$$
\mathbf{J}_{7 \times 13}=\left[\begin{array}{ccccc}
\mathbf{O}_{3 \times 3} & \mathbf{I}_{3} & \mathbf{O}_{3 \times 1} & \mathbf{O}_{3 \times 3} & \mathbf{O}_{3 \times 3} \\
\mathbf{j}_{21} & \mathbf{j}_{22} & \dot{\boldsymbol{v}}_{\dot{\gamma}} & \mathbf{j}_{24} & \mathbf{j}_{25} \\
\mathbf{j}_{31} & \mathbf{j}_{32} & \ddot{\gamma}_{\dot{\gamma}} & \mathbf{O}_{1 \times 3} & -\mathbf{j}_{32}
\end{array}\right]
$$

Where, $\mathbf{j}_{22}, \dot{\boldsymbol{v}}_{\dot{\gamma}}$, and $\mathbf{j}_{25}$ are the partial derivatives of the projectile acceleration with respect to the its velocity, spin rate and wind velocity respectively; $\mathbf{j}_{32}$ and $\ddot{\gamma}_{\dot{\gamma}}$ the partial derivatives of the rate of change of projectile spin rate with respect to its velocity and spin rate respectively [21]. The partial derivatives of the projectile velocity vector $\mathbf{j}_{21}$, spin rate $\mathbf{j}_{31}$ and the repose $\mathbf{j}_{41}$ angle with respect to position vector are given by

$$
\begin{gathered}
\mathbf{j}_{21}=\left[-a_{1}\left|\boldsymbol{v}_{r}\right|\left(\rho C_{x}\right)_{H} \boldsymbol{v}_{r}+a_{1}\left|\boldsymbol{v}_{r}\right|^{2}\left(\rho C_{y}^{\prime}\right)_{H} \boldsymbol{\alpha}_{\mathrm{R}}-a_{2} \dot{\gamma}\left(\rho C_{z}^{\prime \prime}\right)_{H}\left[\boldsymbol{v}_{r} \times \boldsymbol{\alpha}_{\mathrm{R}}\right]\right] \cdot \frac{\partial H}{\partial \mathbf{X}^{T}} \\
\mathbf{j}_{31}=-b_{1}\left|\boldsymbol{v}_{r}\right| \dot{\gamma}\left(\rho m_{x D}^{\prime}\right)_{H} \frac{\partial H}{\partial \mathbf{X}^{T}} \\
\mathbf{j}_{41}=\frac{-b_{4} \dot{\gamma}\left(\rho \cdot m_{z}^{\prime}\right)_{H}}{\left(\rho . m_{z}^{\prime}\right)^{2}\left|\boldsymbol{v}_{r}\right|^{4}}\left[\mathbf{g} \times \boldsymbol{v}_{r}\right] \cdot \frac{\partial H}{\partial \mathbf{X}^{T}}
\end{gathered}
$$

Where, $b_{4}=\left(d / b_{1}\right)$, and $\left(\rho C_{i}\right)_{H}=\partial\left(\rho C_{i}\right) / \partial H=\rho_{H} C_{i}+\rho\left(\partial C_{i} / \partial M\right) M_{H}$

The computations error due to the change in wind and repose angle respectively are given by

$$
\mathbf{J}_{\Delta \boldsymbol{w}}=\left[\begin{array}{ccc}
\mathbf{I}_{3} & \mathbf{O}_{3 \times 3} & \mathbf{O}_{3 \times 1} \\
\mathbf{O}_{3 \times 3} & \mathbf{I}_{3} & \mathbf{O}_{3 \times 1} \\
\mathbf{O}_{1 \times 3} & \mathbf{O}_{1 \times 3} & 1 \\
\mathbf{O}_{3 \times 3} & \mathbf{O}_{3 \times 3} & \mathbf{O}_{3 \times 1} \\
\boldsymbol{w}_{\mathbf{X}} & \dot{\boldsymbol{w}}_{\boldsymbol{v}} \Delta t / 2 & \mathbf{O}_{3 \times 1}
\end{array}\right]
$$

Where,

$$
\mathbf{J}_{\Delta \boldsymbol{\alpha}}=\left[\begin{array}{ccccc}
\mathbf{I}_{3} & \mathbf{O}_{3 \times 3} & \mathbf{O}_{3 \times 1} & \mathbf{O}_{3 \times 3} & \mathbf{O}_{3 \times 3} \\
\mathbf{O}_{3 \times 3} & \mathbf{I}_{3} & \mathbf{O}_{3 \times 1} & \mathbf{O}_{3 \times 3} & \mathbf{O}_{3 \times 3} \\
\mathbf{O}_{1 \times 3} & \mathbf{O}_{1 \times 3} & \mathbf{1} & \mathbf{O}_{1 \times 3} & \mathbf{O}_{1 \times 3} \\
\mathbf{j}_{41} & \mathbf{j}_{42} & \mathbf{j}_{43} & \mathbf{O}_{3 \times 3} & -\mathbf{j}_{42} \\
\mathbf{O}_{3 \times 3} & \mathbf{O}_{3 \times 3} & \mathbf{O}_{3 \times 1} & \mathbf{O}_{3 \times 3} & \mathbf{I}_{3}
\end{array}\right]
$$

Where, $\dot{\boldsymbol{w}}_{\boldsymbol{v}}$ is the change in wind velocity vector with respect to the projectile position; $\mathbf{j}_{42}$ and $\mathbf{j}_{43}$ are the first order projectile angle of repose derivative w.r.t. projectile velocity and spin rate respectively [21]. 
Finally, the elements of the transfer matrix $\Phi$, which maps the state error $\Delta \mathbf{S}^{t_{i}}$ into the state error $\Delta \mathbf{S}^{t_{i+1}}$ is given by

$$
\boldsymbol{\Phi}^{t_{i}}=\left[\begin{array}{ccc}
\mathbf{I}_{3} & \mathbf{I}_{3} \Delta t & \mathbf{O}_{3 \times 1} \\
\boldsymbol{\Delta}_{21} & \boldsymbol{\Delta}_{22} & \boldsymbol{\Delta}_{23} \\
\boldsymbol{\Delta}_{31} & \boldsymbol{\Delta}_{32} & \boldsymbol{\Delta}_{33}
\end{array}\right]
$$

Where,

$$
\begin{gathered}
\Delta_{21}=\left[\mathbf{j}_{21}+\mathbf{j}_{24} \cdot \mathbf{j}_{41}+\left[\mathbf{j}_{25}-\mathbf{j}_{24} \cdot \mathbf{j}_{42}\right] \boldsymbol{w}_{\mathbf{X}}\right] \Delta t \\
\Delta_{22}=\mathbf{I}_{3}+\left[\mathbf{j}_{22}+\mathbf{j}_{24} \cdot \mathbf{j}_{42}\right] \Delta t+\left[\mathbf{j}_{25}-\mathbf{j}_{24} \cdot \mathbf{j}_{42}\right] \dot{\boldsymbol{w}}_{\boldsymbol{v}} \Delta t^{2} / 2 \\
\Delta_{23}=\left[\dot{\boldsymbol{v}}_{\dot{\gamma}}+\mathbf{j}_{24} \cdot \mathbf{j}_{43}\right] \Delta t \\
\Delta_{31}=\left[\mathbf{j}_{31}-\mathbf{j}_{32} \boldsymbol{w}_{\mathbf{X}}\right] \Delta t \\
\Delta_{32}=\mathbf{j}_{32} \Delta t-\mathbf{j}_{32} \dot{\boldsymbol{w}}_{\boldsymbol{v}} \Delta t^{2} / 2 \\
\Delta_{33}=1+\ddot{\gamma}_{\dot{\gamma}} \Delta t
\end{gathered}
$$

The proposed algorithm is dealing with the correction procedure as two one-dimensional problems, in which quadrant elevation angle QE and azimuth angle AZ are obtained separately as illustrated below;

\subsection{Quadrant elevation angle determination}

During projectile launch angle correction process, the first angle to be estimated is $\mathrm{QE}=\theta_{c}$, where the projectile maximum range is achieved by $\left(\theta \approx 45^{\circ}\right)$. For a certain projectile muzzle velocity, there are two different $\mathrm{QE}$ give the same range with different flight time and firing QE angle (low and high firing angles). During searching, the low firing angle is used ( $\theta \leq$ $45^{\circ}$ ), where the flight time is decreased always and hence, reduce the wind effect.

Traditionally, the QE can be determined and corrected using firing tables lookup search methods. But building precise firing tables need a lot of firing test at to cover different firing conditions. Therefore, an iterative Newton-Raphson method will be used in this research as illustrated below

$$
\theta_{j+1}= \begin{cases}\theta_{j}-\frac{X_{\mathrm{P}}-\text { Range }}{\left(f_{1}^{\prime}+f_{2}^{\prime}\right) / 2} & \left.X_{\mathrm{P}}\right|_{-\Delta \theta} \leq \text { Range } \leq\left. X_{\mathrm{P}}\right|_{+\Delta \theta} \\ \left(\theta_{j}+\Delta \theta\right)-\frac{\left.X_{\mathrm{P}}\right|_{+\Delta \theta}-\text { Range }}{2 f_{1}^{\prime}-f_{2}^{\prime}} & \text { Range }>\left.X_{\mathrm{P}}\right|_{+\Delta \theta} \\ \left(\theta_{j}-\Delta \theta\right)-\frac{\left.X_{\mathrm{P}}\right|_{-\Delta \theta}-\text { Range }}{2 f_{2}^{\prime}-f_{1}^{\prime}} & \text { Range }<\left.X_{\mathrm{P}}\right|_{-\Delta \theta}\end{cases}
$$

Where, $j$ is the iteration number, and the corresponding range derivative w.r.t the projectile $\mathrm{QE}$ is given by 


$$
f_{1}^{\prime}=\frac{\left.X_{\mathrm{P}}\right|_{+\Delta \theta}-X_{\mathrm{P}}}{\Delta \theta}, \quad f_{2}^{\prime}=\frac{X_{\mathrm{P}}-\left.X_{\mathrm{P}}\right|_{-\Delta \theta}}{\Delta \theta}
$$

\subsection{Azimuth angle determination}

Finding the projectile launch azimuth angle $\psi$ is much easier and faster than finding QE. Then azimuth angle can be adjusted by the direction error of the impact point of the final iteration as follows,

$$
\begin{gathered}
\psi_{j+1}=\psi_{j}+\Delta \psi \\
\Delta \psi=\tan ^{-1}\left(\frac{Y_{\mathrm{P}}-Y_{\mathrm{T}}}{X_{\mathrm{P}}}\right)
\end{gathered}
$$

\section{ALGORITHM VALIDATION}

The validation process of the proposed algorithm is divided into two phases, (1) the validation of the estimation accuracy of the projectile range derivative w.r.t the QE $\left(\partial X_{\mathrm{P}} / \partial \theta\right),(2)$ the validation of the overall correction algorithm on the mean trajectory impact point w.r.t the reference trajectory.

Firstly, the projectile range derivative w.r.t the $\mathrm{QE}\left(\partial X_{\mathrm{P}} / \partial \theta\right)$ is estimated in order to compute the amount of QE needed during correction process. Then, the upper and lower bound trajectories will be computed, where these bounds are $\pm \Delta \theta$ from the reference trajectory QE.

Therefore, a number of ballistic trajectories for $105 \mathrm{~mm}-\mathrm{HE}$ projectile is generated as shown in Fig. 2, where $\partial X_{\mathrm{P}} / \partial \theta$ is always a function of QE. The projectile mass properties and aerodynamic coefficients have been illustrated in [22]. All trajectories are generated using 4DOF model with $\theta=10,20 \ldots 70^{\circ}$ and $\psi=0^{\circ}$, where the projectile is traveling through wind profile shown in Fig. 3. The trajectory data frequency is assumed to be $0.001 \mathrm{~s}$ and the $\Delta \theta=1^{\circ}$.

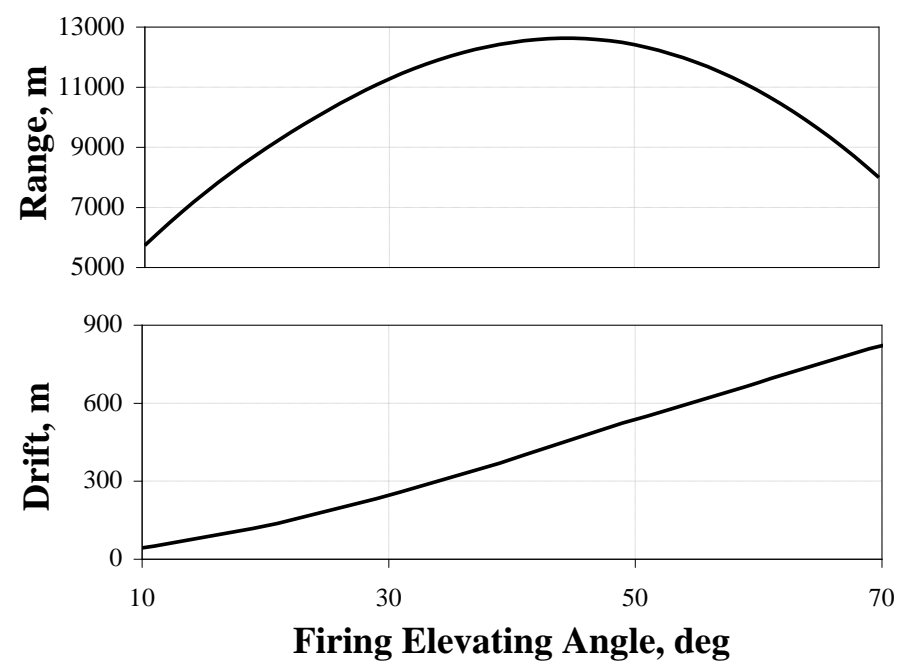

Fig. 2 The relation between the projectile QE and corresponding range and drift 


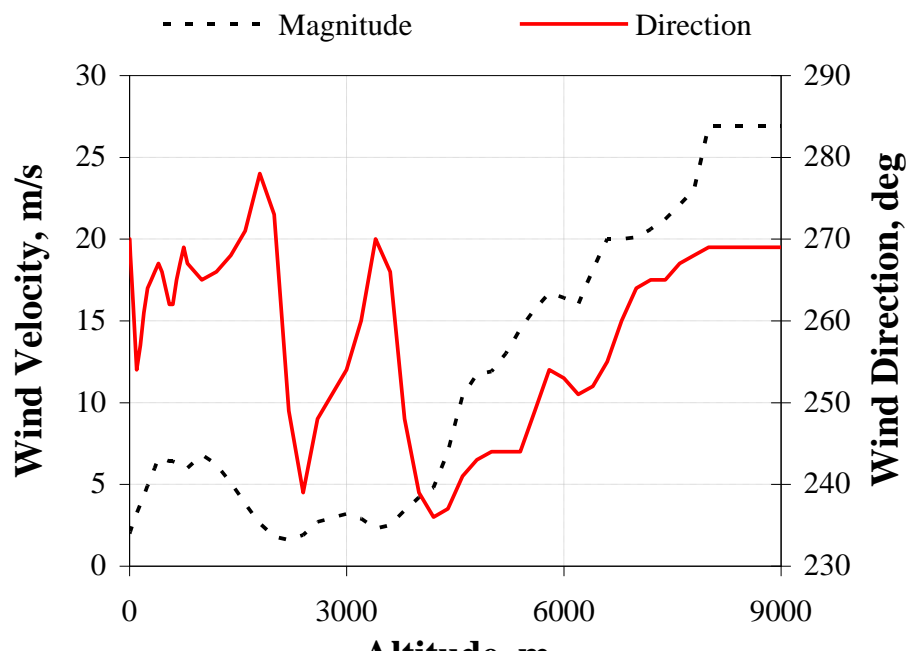

Altitude, $\mathbf{m}$

Fig. 3 Wind velocity and direction versus altitude

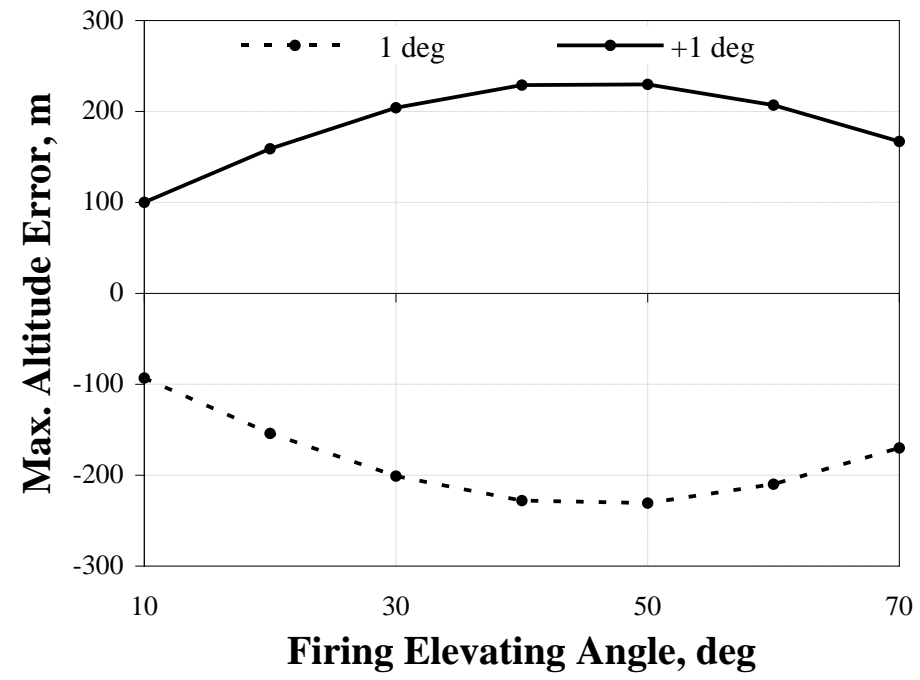

Fig. 4 The maximum altitude difference between bound trajectories and reference

(a)

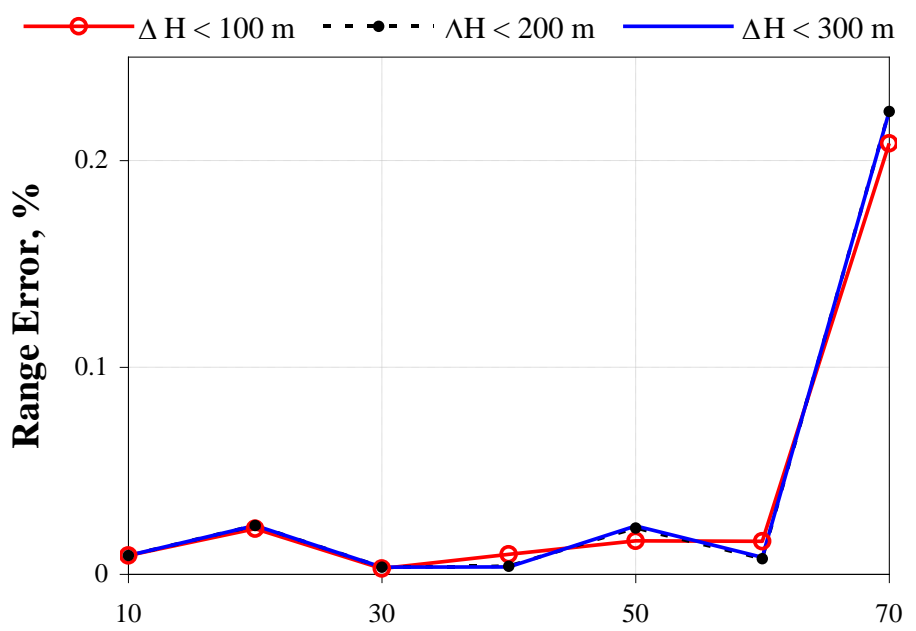

Firing Elevating Angle, deg 
(b)

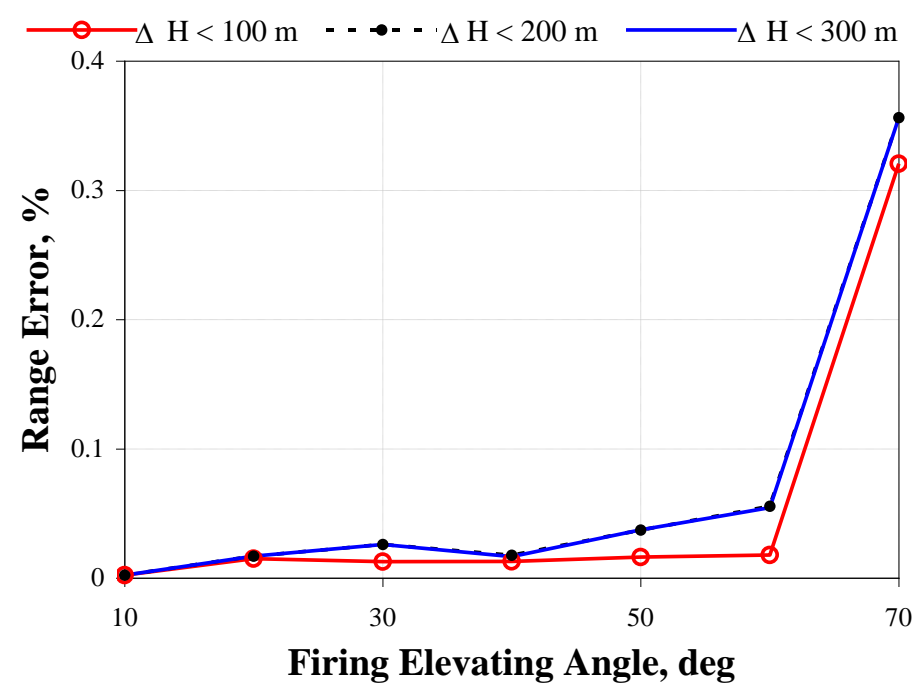

Fig. 5 The range error versus the projectile QE for a) $\Delta \theta=-1^{\circ}$, b) $\Delta \theta=+1^{\circ}$

(a)

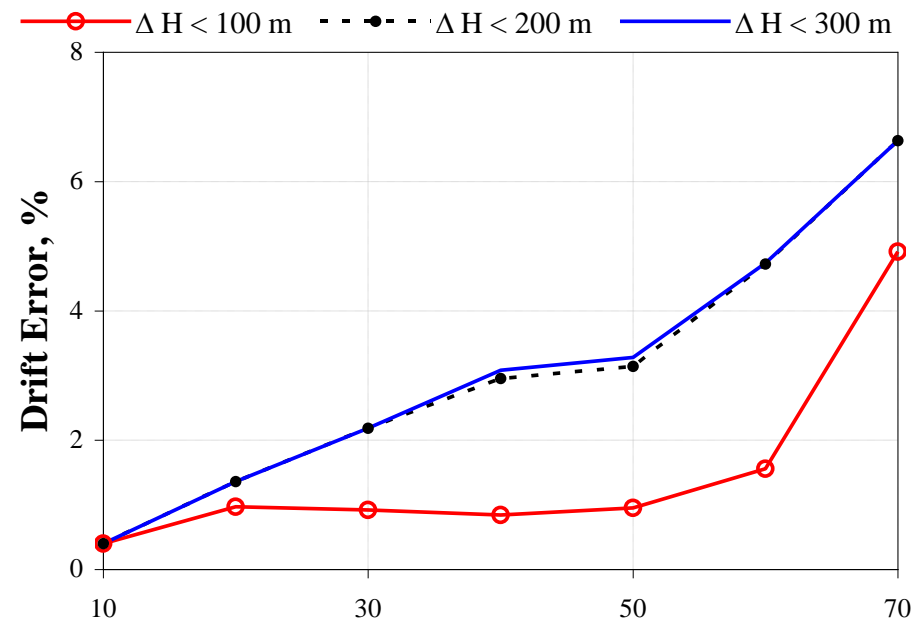

Firing Elevating Angle, deg

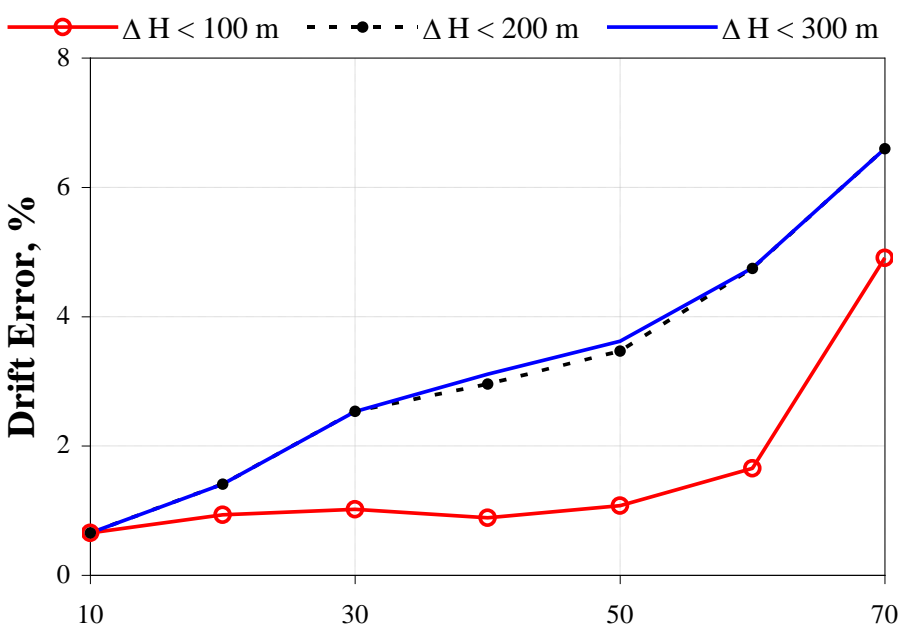

Firing Elevating Angle, deg

Fig. 6 The drift error versus the projectile QE for a) $\Delta \theta=-1^{\circ}$, b) $\Delta \theta=+1^{\circ}$ 
The accuracy of the state correction matrix $\boldsymbol{\Phi}^{t_{i}}$ is strongly affected by some parameters which are deviated from the reference trajectory such as the altitude and repose angle. Therefore, a comparative study has been done between different altitude bound $\Delta H$, where if the altitude error for the estimated trajectory is greater than $\Delta H$, Eq. (11) will be terminated and the rest of trajectory will be computed from scratch using DTTM-4DOF model.

Figures 5 and 6 show the relative range and drift accuracy respectively w.r.t the reference, where as the altitude difference $(\Delta H)$ between the reference state and the estimated state increased, the error will be increased. It can be concluded that the effect of changing $\Delta H$ has a negligible error on the projectile range error for low $\mathrm{QE}$ ( $\theta \leq$ $45^{\circ}$ ). But in case of high QE, the range and drift errors can not be neglected even at $\Delta H<100 \mathrm{~m}$ due to large repose angle at high QE as shown in Fig 7. Therefore, the proposed algorithm will be used only for low QE to have best performance. As shown in Fig 8, the time consumed during the computation of the reference trajectory $\mathrm{QE}=\theta$ and the two other trajectories $\mathrm{QE}=\theta \pm \Delta \theta$ using 4-DOF model decreased by more than $60 \%$ using the state correction matrix $\boldsymbol{\Phi}^{t_{i}}$ for $(\Delta H<300 \mathrm{~m})$ due to decreasing number of operations performed during each time step by directly obtain two trajectories using the correction matrix multiplied by the reference trajectory instead of computing from scratch using 4-DOF model.
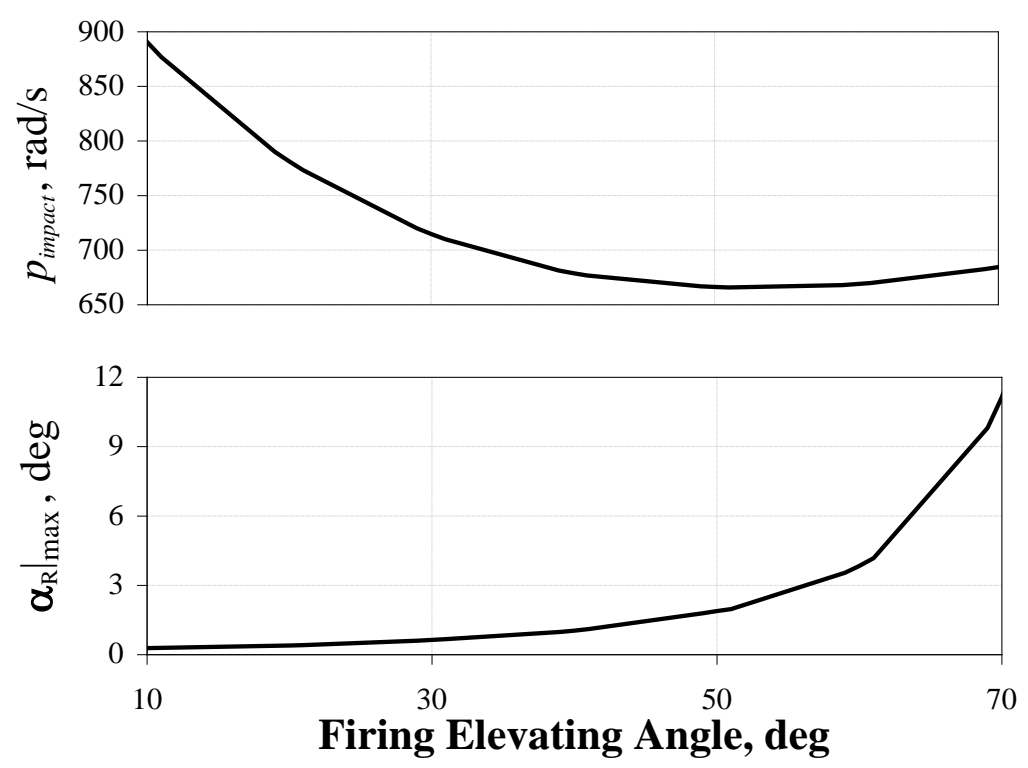

Fig. 7 The projectile impact spin rate $\dot{\gamma}$ and max. repose angle $\alpha$ versus QE

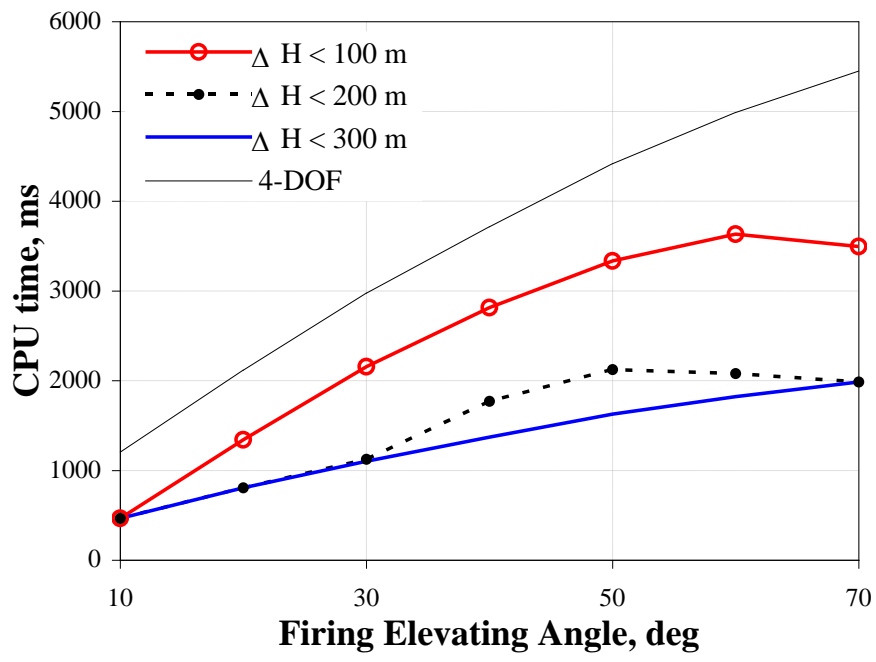

Fig. 8 The CPU time study 
Table 1 Trajectory Correction

\begin{tabular}{lllll}
\hline \multirow{2}{*}{ Case No. } & \multicolumn{2}{l}{ Impact Point (real wind) } & \multicolumn{2}{l}{ Target Point (zero wind) } \\
\cline { 2 - 5 } & Range, $\mathrm{m}$ & Drift, $\mathrm{m}$ & Range, $\mathrm{m}$ & Drift, $\mathrm{m}$ \\
\hline 1 & 12075.19 & 129.6152 & 12159.26 & 134.5334 \\
\hline \multirow{2}{*}{2} & \multirow{2}{*}{20047.84} & 594.5224 & 19047.84 & 494.5224 \\
\cline { 3 - 5 } & & & 19547.84 & 550.5224 \\
\hline 3 & 21558.97 & 853.0912 & 22260.67 & 628.9069 \\
\hline
\end{tabular}

Secondly, using a spin-stabilized $155 \mathrm{~mm}-\mathrm{M} 107$ projectile [23], a complete usage of the proposed correction algorithm is done for different cases as mentioned in Table 1 to investigate the solution convergence during iterations. In case of firing at standard conditions with zero wind, the target point in case $1,2-\mathrm{a}$, and 3 are obtained using $\mathrm{QE}=10^{\circ}, 30^{\circ}$ and $40^{\circ}$ respectively as listed in Table 2 . But using non-standard conditions with wind profile shown in Fig. 3, the impact points are deviated from the desired targets and it's hard to obtain the corresponding firing angle to hit the target point with an appropriate accuracy.

Results of the case-1 are listed in Table 2. These results show the speed of the proposed algorithm, where the required two dimensional firing angle can be estimated just in one iteration with miss distance $\varepsilon \approx 1$, where the projectile range is strongly affected by a small variation in firing $\mathrm{QE}$.

Case-2 examines the effect of miss distance on the number of iterations needed to improve the estimated firing angle accuracy.

Table 3 shows that the adjustments made in the gun azimuth $\psi$ is always made after estimating the gun elevation $\theta$, where the effect of range variation during iteration process has a strong impact on the corresponding azimuth $\psi$ as mentioned in Fig 2. However, the effect of azimuth variation $\Delta \psi$ on the resultant range is very small and can be neglected. As the projectile miss-distance $\varepsilon$ increases, the solution number of iterations is also increases.

Case- 3 is used to test the proposed correction algorithm near the maximum firing elevating angle $40^{\circ} \leq \theta \leq 45^{\circ}$, where the projectile range variation relative to firing QE is very small compared to cases 1 and 2. The results for this case are listed in Table 4. These results show the robustness of the proposed algorithm. A stopping condition is applied to the proposed algorithm, where the maximum fringing elevating angle can reached is $\theta=45^{\circ}$. As expected, the solution converged and discontinued.

Table 2 Case-1 simulation results

\begin{tabular}{llllll}
\hline Iteration & $\varepsilon_{\text {range }}, \mathrm{m}$ & $\varepsilon_{\text {drift }}, \mathrm{m}$ & Miss Distance, $\mathrm{m}$ & $\theta, \mathrm{deg}$ & $\psi, \mathrm{deg}$ \\
\hline 0 & -84.0672 & -4.91824 & 84.21099 & 10 & 0 \\
\hline 1 & -0.71067 & 0.746387 & 1.030605 & 10.13755 & 0.013221 \\
\hline
\end{tabular}




\begin{tabular}{llllll}
\hline Iteration & $\varepsilon_{\text {range }}, \mathrm{m}$ & $\varepsilon_{\text {drift }}, \mathrm{m}$ & Miss Distance, $\mathrm{m}$ & $\theta, \mathrm{deg}$ & $\psi, \mathrm{deg}$ \\
\hline 0 & 1000 & 100 & 1004.988 & 30 & 0 \\
\hline 1 & -325.616 & -12.9003 & 325.8712 & 25.30706 & 0 \\
\hline 2 & -3.33624 & 17.25996 & 17.57944 & 26.32621 & 0 \\
\hline 3 & 0.574426 & -2.14406 & 2.219676 & 26.33711 & -0.05288 \\
\hline
\end{tabular}

Table 4 Case-3 simulation results

\begin{tabular}{llllll}
\hline Iteration & $\varepsilon_{\text {range }}, \mathrm{m}$ & $\varepsilon_{\text {drift }}, \mathrm{m}$ & Miss Distance, $\mathrm{m}$ & $\theta, \mathrm{deg}$ & $\psi, \mathrm{deg}$ \\
\hline 0 & -701.698 & -99.6278 & 708.7352 & 40 & 0 \\
\hline 1 & -473.171 & -9.719 & 473.2711 & 45 & -0.200013 \\
\hline
\end{tabular}

\section{Conclusion:}

A correction algorithm was developed to accurately estimate the projectile two dimensional firing angles required to impact a desired target, given the projectile launch parameters uncertainties, the current meteorological data, and launch and targeted impact locations. A limitation has been placed to the proposed algorithm, where the maximum firing elevating angle available is $\theta=45^{\circ}$. A state correction matrix was deduced using the discrete time transfer matrix for modified point mass trajectory model DTTM-4DOF. The main advantage of the proposed algorithm is decreasing the number of operations performed during each firing angle and hence; the computation time needed for estimating the projectile impact point and the corresponding impact range derivative relative to QE by approximately $60 \%$ than using traditional methods in literature. Results demonstrate that the solution of proposed correction algorithm is always converged with high accuracy.

\section{References}

[1] Shi W. X., Qian J.P. and Fu C., etc. Simulating the Effectiveness of Naval Gun Fire on the Sea by Monte Carlo Method. Journal of Sichuan Ordnance, 2011, 32(6), 14-17.

[2] Dickinson E. R. The production of firing tables for cannon artillery. BRL Report 1371, Ballistic Research Laboratory, MD, USA, 1967.

[3] Chusilp P., Charubhun W. and Nutkumhang N. Investigating an iterative method to compute firing angles for artillery projectiles. The 2012 IEEE/ASME International conference on Advanced Intelligent Mechatronics, Kaohsiung, Taiwan, July 11-14, 2012, 940-945.

[4] Hansen B.R., Aytarortac S. and Sowa A.J. NATO testing in Turkey show benefit of meteorological forecast data to indirect fire support, $24^{\text {th }}$ International Symposium on Ballistics, New Orleans, LA, USA, September 22-26, 2008.

[5] Jameson T.C., Luces S.A. and Knapp D. Comparisons of ballistic trajectory simulations using artillery meteorological messages derived from local balloon data and battlescale forecast model data for the 1998 SADARM IOT\&E firings. ARL-TR1018, Army Research Laboratory, MD, USA, 2001.

[6] Matts J.A. and McCoy D.H. A graphical firing table model and a comparison of the accuracy of three utilization schemes. BRL Report No. 2035, Ballistic Research Laboratory, MD, USA, 1970.

[7] Liu Y. and Yang Q. A Mathematical Model of Determining Firing Data By Computer. 
Journal of Ballistics, 1996, 8(3), 66-68.

[8] Xue X. and Liu X. Weighted Function Method For Exterior Ballistic Model In Fire Control System. Acta Armamentarii, 1997, 18(3), 234-238.

[9] Zhou Q., Yu L. and Giu X. Research on Real-Time Ballistic for Battle Vehicles. Fire Control and Command Control, 2001, 26(4), 15-18.

[10] Pan H., Hu J., Yang S. and Wang D. Method to Calculate the Ship-borne Gun Fire Data Against Shore. Fire Control and Command Control, 2001, 26(4), 51-53.

[11] Pan. H., Hu J., Yang S. and Wang D. Model For Finding Firing Data Of Ship Borne Gun Weapon System By Solving External Ballistic Equations. Acta Armamentarii, 2003, 24(1), 23-26.

[12] Harlin W. J. and Cicci D. A. Ballistic missile trajectory prediction using a state transition matrix. Applied Mathematics and Computation, 2007,188(2),1832-1847.

[13] Yu J., Zhang A. and Liu W. Method of Calculating Ahead Hitting Position of Antiaircraft Gun and Its Accuracy Simulation. Computer Simulation, 2011, 28(5), 21 25 .

[14] Chusilp P., Charubhun W. and Ridluan A. Developing firing table software for artillery projectile using iterative search and 6-DOF trajectory model. In the Second TSME International Conference on Mechanical Engineering, Krabi, Thailand, October 19-21, 2011.

[15] Sowa A.J. NATO shareable software developing into true suite supporting national operational fire control systems. $24^{\text {th }}$ International Symposium on Ballistics, New Orleans, LA, USA, September 22-26, 2008.

[16] Lieske R. F. and Reiter M. L. Equations of Motion for a Modified Point Mass Trajectory. U.S. Army Ballistic Research Laboratory, U.S. Army, Aberdeen Proving Ground, Md., 1966, Rep. No. R1314 (AD 485869).

[17] Zipfel P. Modeling and Simulation of Aerospace Vehicle Dynamics, 2nd edition, AIAA, USA, 2007.

[18] Ortac S.A., Durak U. and Kutluay U., etc. NABK based next generation ballistic table toolkit. $23^{\text {rd }}$ International Symposium on Ballistics, Tarragona, Spain, April 16-20, 2007.

[19] Zhang X. Calculating Shoot Parameter by Neural Net. Shanxi Electronic Technology, 2005, 2005(2), 33-35.

[20] Khalil M., Rui X. and Zha Q., etc. Projectile Impact Point Prediction Based on SelfPropelled Artillery Dynamics and Doppler Radar Measurements. Advances in Mechanical Engineering, 2013, (2013), Article ID 153913, 12 pages.

[21] Khalil M., Rui X. and Zha Q., etc. Projectile Impact Point Prediction Based on SelfPropelled Artillery Dynamics and Doppler Radar Measurements. Advances in Mechanical Engineering, 2013, (2013), Article ID 153913, 12 pages.

[22] McCoy R. L. Modern Exterior Ballistics: The Launch and Fight Dynamics of Symmetric Projectiles. China, Schiffer, 1999.

[23] Khalil M., Abdalla H. and Kamal O. Dispersion Analysis for Spinning Artillery Projectile. Proceeding of the $13^{\text {th }}$ International conference on Aerospace Science \& Aviation Technology, Cairo, Egypt, May 26 -28, 2009. 